
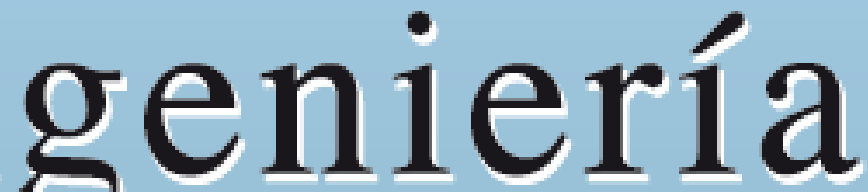

Revista de la Universidad de Costa Rica ENEROIDICIEMBRE 2002 - VOLUMEN 12 - $\mathrm{N}^{\circ} 1$ y 2

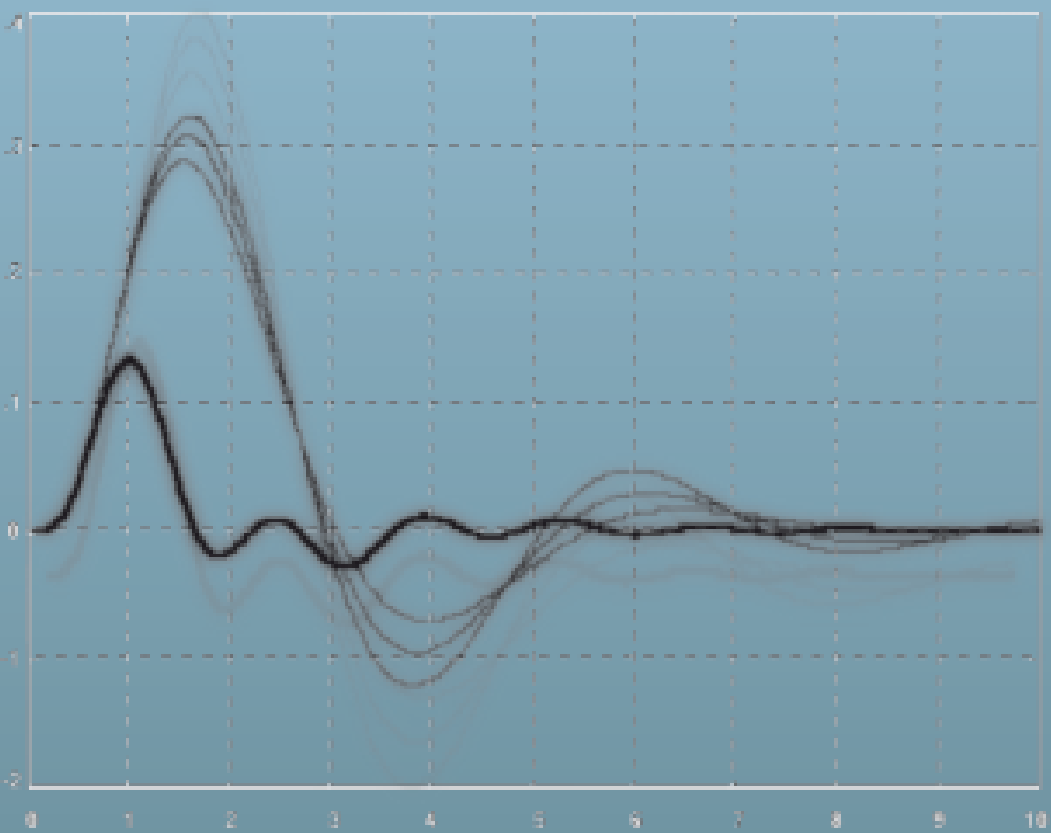




\title{
MÉTODOS DE SINTONIZACIÓN DE CONTROLADORES PID QUE OPERAN COMO REGULADORES
}

Víctor M. Alfaro Ruíz

\section{Resumen}

Se presentan varios de los métodos de sintonización de controladores PID para lazos de control que operan como reguladores. Mediante pruebas de simulación digital se compara el desempeño de los diferentes métodos y se determina la bondad de los mismos con base en criterios de comportamiento y robustez. Finalmente, se ofrecen recomendaciones sobre la utilización de los métodos probados.

Palabras claves: controladores PID, sintonización, reguladores.

\begin{abstract}
Several methods for tuning PID controllers working as regulators are presented. The behavior and goodness of the tuning methods are compared by digital simulation using several performance and robustness criteria. Recommendations for the use of the tested tuning methods are given based on the performance requirements.
\end{abstract}

Keywords: PID controllers, tuning rulers, load changes.

\section{INTRODUCCIÓN}

La sintonización de los controladores Proporcional - Integral - Derivativo o simplemente controladores $\boldsymbol{P I D}$, consiste en la determinación del ajuste de sus parámetros $\left(K_{c}, T_{i}, T_{d}\right)$, para lograr un comportamiento del sistema de control aceptable y robusto de conformidad con algún criterio de desempeño establecido.

Para poder realizar la sintonización de los controladores, primero debe identificarse la dinámica del proceso, y a partir de ésta determinar los parámetros del controlador utilizando el método de sintonización seleccionado.

Antes de revisar algunas de las técnicas de sintonización disponibles, se establecerán los diferentes tipos de funcionamiento que pueden presentarse en un lazo de control realimentado, en el cual hay dos entradas - el valor deseado r(t) y la perturbación $z(t)$ - y una salida - la señal realimentada $y(t)$-, como se muestra en la Figura 1.

Si se considera las variables en el dominio de la variable compleja $s$ y se define $G c(s)$ y $G p(s)$ como las funciones de transferencia del controlador y de la planta respectivamente, a partir del diagrama de bloques del sistema de control de lazo cerrado, se obtiene que la señal realimentada, representación de la variable controlada, está dada por:

$y(s)=\frac{G_{c}(s) G_{p}(s)}{1+G_{c}(s) G_{p}(s)} r(s)+\frac{G_{p}(s)}{1+G_{c}(s) G_{p}(s)} z(s)$

Se debe considerar, entonces, dos posibles condiciones de operación del sistema de control, en primer lugar:

- Servomecanismo $(\mathrm{z}=0)$

$y(s)=\frac{G_{c}(s) G_{p}(s)}{1+G_{c}(s) G_{p}(s)} r(s)$

Esta condición requiere un buen seguimiento del valor deseado. En segundo lugar:

- Regulador $(\mathrm{r}=0)$

$y(s)=\frac{G_{p}(s)}{1+G_{c}(s) G_{p}(s)} z(s)$

en donde lo importante es la insensibilidad a las perturbaciones. 


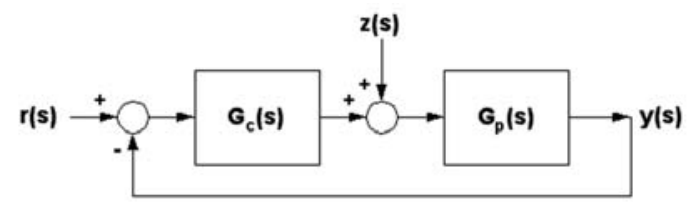

Figura 1. Sistema de control realimentado.

Dado que los numeradores de las funciones de transferencia de lazo cerrado (2) y (3) son diferentes, no necesariamente un buen ajuste del controlador para operar como servomecanismo, proveerá un buen funcionamiento como regulador, razón por la cual se han desarrollado procedimientos de sintonización para ambos tipos de operación.

En la industria de procesos, la mayor parte de los controladores se utilizan para responder a un cambio en la perturbación, y se requiere de una buena regulación para seguir el cambio en el valor deseado, con excepción de los controladores esclavos en los sistemas de control en cascada.

Si el controlador se ha sintonizado para lograr una buena respuesta en un cambio en el valor deseado, no eliminará las perturbaciones en forma efectiva, si el sistema contiene un integrador o si su constante de tiempo es grande.

Es importante, entonces, determinar los requisitos de funcionamiento del lazo de control para seleccionar el procedimiento de sintonización adecuado.

El desarrollo de los métodos de sintonización ha sido extenso desde que Ziegler y Nichols propusieron su procedimiento en 1942. Normalmente, se dividen en métodos de lazo abierto y métodos de lazo cerrado.

\section{- Métodos de lazo abierto}

El controlador cuando está instalado operará manualmente. Produciendo un cambio escalón a la salida del controlador se obtiene la curva de reacción del proceso, a partir de la cual se identifica un modelo para el mismo, usualmente de primer orden más tiempo muerto. Este modelo es la base para la determinación de los parámetros del controlador.

\section{- Métodos de lazo cerrado}

El controlador opera automáticamente produciendo un cambio en el valor deseado se obtiene información del comportamiento dinámico del sistema para identificar un modelo de orden reducido para el proceso, o de las características de la oscilación sostenida del mismo, para utilizarla en el cálculo de los parámetros del controlador.

Se restringirá la presentación de los métodos de sintonización y de los resultados de la comparación, a aquéllos desarrollados para los controladores PID que operan como reguladores y utilizan un modelo del proceso. En particular, nos referiremos a los siguientes métodos por ser los más empleados:

- Ziegler y Nichols

- Cohen y Coon

- López et al.

- Kaya y Sheib

- $\quad$ Sung et al.

O'Dwyer resume una gran variedad de procedimientos de sintonización para controladores $P I^{[12]}$ y $P I D^{[13]}$ más.

\section{CONTROLADORES}

Los controladores PID considerados por los autores de los métodos de sintonización, tienen algunas de las siguientes funciones de transferencia ${ }^{[1]}$ :

- Controlador PID-Ideal

$u(s)=K_{C}\left[1+\frac{1}{T_{i} s}+\frac{T_{d} s}{1+\tau_{f} s}\right](r(s)-y(s)$

- Controlador PID-Serie (Interactuante)

$u(s)=K_{C}^{\prime}\left[1+\frac{1}{T_{i}^{\prime} s}\right]\left[1+\frac{T_{d}^{\prime} s}{1+\tau_{f} s}\right](r(s)-y(s))$ 
- Controlador PID-Industrial

$u(s)=K_{C}^{\prime}\left[1+\frac{1}{T_{i}^{\prime} s}\right]\left[r(s)+\frac{T_{d}^{\prime} s}{1+\tau_{f} s} y(s)\right]$

Si el controlador PID, que se desea sintonizar, no es del mismo tipo que el supuesto en el método de sintonización a emplear, deberá realizarse, la conversión de parámetros necesaria.

\section{MÉTODOS DE SINTONIZACIÓN DE LAZO ABIERTO}

\subsection{Modelos}

Los procedimientos de sintonización de lazo abierto utilizan un modelo de la planta que se obtiene, generalmente, a partir de la curva de reacción del proceso, son:

- $\quad$ Primer orden más tiempo muerto

$G_{p}(s)=\frac{k_{p} e^{-t_{m} s}}{\tau s+1}$

- $\quad$ Polo doble más tiempo muerto

$G_{p}(s)=\frac{k_{p} e^{-t_{m} s}}{(\tau s+1)^{2}}$

- $\quad$ Segundo orden más tiempo muerto

$G_{p}(s)=\frac{k_{p} e^{-t_{m} s}}{\left(\tau_{1} s+1\right)\left(\tau_{2} s+1\right)}$

$G_{p}(s)=\frac{k_{p} e^{-t_{m} s}}{\tau^{2} s^{2}+2 \varsigma \tau s+1}$

Alfaro $^{[2,3]}$ presenta y compara algunas de las técnicas de identificación de lazo abierto y lazo cerrado más utilizadas para la obtención de estos modelos.

\subsection{Método de Ziegler y Nichols}

El primer procedimiento sistematizado para el cálculo de los parámetros de un controlador PID fue desarrollado por Ziegler y Nichols ${ }^{[18]}$. El criterio de desempeño que seleccionaron fue el de un decaimiento de $1 / 4$, o sea que el error decae en la cuarta parte de un periodo de oscilación.

Las ecuaciones fueron determinadas de forma empírica a partir de pruebas realizadas en el laboratorio con diferentes procesos, y están basadas en un modelo de primer orden más tiempo muerto identificado por el método de la tangente, para un funcionamiento del lazo de control como regulador con un controlador PID-Ideal.

En cuanto al tipo de controlador, si bien la gran mayoría de los autores indican que este método es para la sintonización de controladores ideales, otros, entre los que destaca Corripio ${ }^{[8]}$, afirman que este método es para los controladores que interactúan.

Las ecuaciones de sintonización de este método $\operatorname{son}^{1}$ :

$$
\begin{gathered}
K_{C}=1.2 \frac{\tau}{k_{p} t_{m}} \quad \text { a } 2.0 \frac{\tau}{k_{p} t_{m}} \\
T_{i}=2 t_{m} \\
T_{d}=0.5 t_{m}
\end{gathered}
$$

\subsection{Método de Cohen y Coon}

En el desarrollo de su método de sintonización Ziegler y Nichols no consideraron que el proceso fuera auto regulado. Cohen y Coon ${ }^{[8]}$ introdujeron, entonces, un índice de auto regulación definido como $\mu=t_{m} / \tau$ y plantearon nuevas ecuaciones de sintonización. Estas se basan en el mejor modelo de primer orden más tiempo muerto que se pueda obtener para lazos de control que funcionan como regulador, con el criterio de desempeño de decaimiento de $1 / 4$ con sobrepaso mínimo, y con mínima área bajo la curva de respuesta, y un controlador PID-Ideal. Las ecuaciones son:

$$
K_{C}=\frac{\tau}{k_{p} t_{m}}\left(\frac{4}{3}+\frac{t_{m}}{4 \tau}\right)
$$




$$
\begin{aligned}
& T_{i}=t_{m}\left(\frac{32+6 t_{m} / \tau}{13+8 t_{m} / \tau}\right) \\
& T_{d}=t_{m}\left(\frac{4}{11+2 t_{m} / \tau}\right)
\end{aligned}
$$

Witt y Waggoner ${ }^{[17]}$ presentan un cálculo refinado de las ecuaciones anteriores.

\subsection{Método de López, Miller, Smith y Murril}

El primer método basado en criterios integrales que presentó ecuaciones para el cálculo de los parámetros del controlador fue desarrollado por López et al. ${ }^{[11]}$ y es conocido como el método de López.

Definiendo una función de costo de la forma

$\Phi=\int_{0}^{\infty} F[e(t), t] d t$

donde $\mathrm{F}$ es una función del error y del tiempo, se obtiene un valor que caracteriza la respuesta del sistema. Entre menor sea el valor de $\Phi$, mejor será el desempeño del sistema de control, por ello, un desempeño óptimo se obtiene cuando $\Phi$ es mínimo.

Como $\Phi$ es una función de los parámetros del controlador $(K c, T i, T d)$, el valor mínimo de $\Phi$ se obtiene resolviendo las siguientes ecuaciones:

$$
\frac{\partial \Phi}{\partial K_{C}}=0, \frac{\partial \Phi}{\partial T_{i}}=0, \frac{\partial \Phi}{\partial T_{d}}=0
$$

Los criterios de desempeño utilizados por López fueron: Integral del error absoluto (IAE), Integral del error absoluto por el tiempo (ITAE) y Integral del error cuadrático (ISE).
La optimización de los criterios de desempeño integrales de López está basada en el mejor modelo de primer orden más tiempo muerto que se pueda obtener, para lazos de control que funcionan como reguladores con un controlador PID-Ideal.

Las ecuaciones de sintonización son:

$$
\begin{aligned}
& K_{c} k_{p}=a\left(\frac{t_{m}}{\tau}\right)^{b} \\
& \frac{T_{i}}{\tau}=\frac{1}{c}\left(\frac{t_{m}}{\tau}\right)^{-d} \\
& \frac{T_{d}}{\tau}=e\left(\frac{t_{m}}{\tau}\right)^{f}
\end{aligned}
$$

Los valores para las constantes a a f para los diferentes criterios se encuentran en el Cuadro 1.

\subsection{Método de Kaya y Sheib}

Mientras López desarrolló el método de sintonización para un controlador PID-Ideal, Kaya y Sheib ${ }^{[10]}$ realizaron lo mismo para controladores que denominaron PID-Clásico (PID-Serie), PIDNo Interactuante (una variación del PID-Paralelo) y PID-Industrial.

El procedimiento de sintonización está basado en el mejor modelo de primer orden más tiempo muerto que se pueda obtener para lazos de control que funcionan como reguladores. El criterio de desempeño corresponde a la minimización de alguno de los criterios integrales y el controlador a uno de los indicados anteriormente.

Las ecuaciones de sintonización son las mismas dadas por López (14) y los valores de los parámetros $a$ a $f$ se muestran en el Cuadro 2. 
Cuadro 1. Constantes para las ecuaciones del método de López

$\begin{array}{ll}\text { IAE } & 1,435 \\ \text { ITAE } & 1,357\end{array}$

ISE $\quad 1,495$

\begin{abstract}
b
\end{abstract}

$-0,921$

$-0,947$

$-0,945$

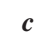

\begin{abstract}
0,878
\end{abstract}
0,842

1,101

$-0,749$

$-0,738$

$-0,771$

0,560 f

1,137

0,995

1,006

Cuadro 2. Constantes para las ecuaciones del método de Kaya y Sheib

\begin{tabular}{lcccccc}
\multicolumn{7}{c}{ Controlador “clásico" - Regulador } \\
& $\boldsymbol{a}$ & $\boldsymbol{b}$ & $\boldsymbol{c}$ & $\boldsymbol{d}$ & $\boldsymbol{e}$ & $\boldsymbol{f}$ \\
IAE & 0,98089 & $-0,76167$ & 0,91032 & $-1,05211$ & 0,59974 & 0,89819 \\
ITAE & 0,77902 & $-1,06401$ & 1,14311 & $-0,70949$ & 0,57137 & 1,03826 \\
ISE & 1,11907 & $-0,89711$ & 0,79870 & $-0,95480$ & 0,54766 & 0,87798
\end{tabular}

$a$

IAE $\quad 1,31509$

ITAE 1,31760

ISE $\quad 1,34660$

$a$

IAE $\quad 0,91000$

ITAE $\quad 0,70580$

ISE $\quad 1,11470$

\section{Controlador "no interactuante" - Regulador}

b

$-0,88260$

$c$

$-0,79370$

$-0,93080$

1,25870

$-1,37560$

0,56550

$-1,42603$

0,49547

0,79715

$-1,25738$

1,65850

Controlador "industrial" - Regulador

b

$-0,79380$

$c$

$-0,88720$

1,01495

\section{d}

$-1,00403$

$-0,99138$

$-0,87530$ $\boldsymbol{e}$

0,54140

0,60006

0,56508
$-0,89920$

0,93240

\section{$f$}

0,45760

0,41932

0,41941

0,56508 


\subsection{Método de Sung, O, Lee, Lee y Yi}

Los métodos de sintonización de controladores que optimizan los criterios integrales, vistos anteriormente, se basan en un modelo de primer orden más tiempo muerto. Sung et al. ${ }^{[15]}$ por su parte, basó su procedimiento de sintonización en un modelo de segundo orden más tiempo muerto para un proceso como el dado por (9b), identificado mediante la realización de una prueba con realimentación por relé, seguida por una con control $P$.

El procedimiento está basado en el mejor modelo de segundo orden más tiempo muerto que se pueda obtener para lazos de control que funcionan como reguladores. El criterio de desempeño corresponde a la minimización de la ITAE y el controlador un PID-Ideal. El ajuste de las ecuaciones la realizaron para $0,05 \leq t_{m} / \tau \leq 2,0$.

Las ecuaciones de sintonización son:

$$
K_{c} k_{p}=-0.67+0.297\left(\frac{t_{m}}{\tau}\right)^{-2.001}+2.189\left(\frac{t_{m}}{\tau}\right)^{-0.766} \varsigma \quad, \frac{t_{m}}{\tau} \leq 0.9
$$

$K_{c} k_{p}=-0.365+0.260\left(\frac{t_{m}}{\tau}-1.4\right)^{2.0}+2.189\left(\frac{t_{m}}{\tau}\right)^{-0.766} \varsigma \quad, \frac{t_{m}}{\tau} \geq 0.9$

$$
\frac{T_{i}}{\tau}=2.212\left(\frac{t_{m}}{\tau}\right)^{0.520}-0.3 \quad, \quad \frac{t_{m}}{\tau}<0.4
$$

$\frac{T_{i}}{\tau}=-90.975+0.91\left(\frac{t_{m}}{\tau}-1.845\right)^{2}+\left[1-E X P\left(\frac{-\varsigma}{0.15+0.33\left(t_{m} / \tau\right)}\right)\right]\left[5.25-0.88\left(\frac{t_{m}}{\tau}-2.8\right)^{2}\right], \frac{t_{m}}{\tau} \geq 0.4$

$$
\frac{\tau}{T_{d}}=-1.9+1.576\left(\frac{t_{m}}{\tau}\right)^{-0530}+\left[1-\operatorname{EXP}\left(\frac{-\varsigma}{-0-15+0.939\left(t_{m} / \tau\right)^{-1.121}}\right)\right]\left[1.45+0.969\left(\frac{t_{m}}{\tau}\right)^{-1.171}\right]
$$




\section{MÉTODOS DE SINTONIZACIÓN DE LAZO CERRADO}

\subsection{Método de Ziegler y Nichols}

Al igual que sucedió con los procedimientos de sintonización basados en la curva de reacción del proceso, el primer procedimiento de sintonización basado en una prueba de lazo cerrado fue propuesto por Ziegler y Nichols, quienes presentaron ambos procedimientos en la misma publicación ${ }^{[18]}$.

Utilizando un controlador puramente proporcional y mediante un proceso iterativo, el procedimiento requiere aumentar paulatinamente la ganancia del mismo hasta lograr que el sistema entre en una oscilación sostenida ante un cambio del escalón en el valor deseado. La ganancia en este punto es la ganancia última $K_{c u}$ y el periodo de la oscilación, el periodo último $T_{u}$.

Para el ajuste proporcional seleccionaron, como se indicó, el decaimiento de 1/4 como un compromiso entre el error permanente y el decaimiento, y encontraron que la ganancia proporcional para un controlador $P$ debería ser la mitad de la ganancia última.

Las ecuaciones de sintonización del controlador PID son:

$$
\begin{aligned}
& K_{c}=0,6 K_{c u} \text { a } 1,0 K_{c u} \\
& T_{i}=0,5 T_{u} \\
& T_{d}=0,125 T_{u}
\end{aligned}
$$

La información última $\left(K_{c u}, T_{u}\right)$ utilizada en las ecuaciones anteriores, también puede ser obtenida mediante una prueba con realimentación con relé como lo propusieron Aström y Hägglund ${ }^{[4]}$.

\subsection{Variaciones del método de Ziegler y Nichols}

No solo por ser el primero sino por que se emplea todavía en su forma original o en alguna de sus variantes, los métodos de sintonización desarrollados por Ziegler y Nichols se han convertido en el patrón en el que por años, se han establecido las comparaciones entre muchos de los métodos de sintonización de controladores.

Por ejemplo, Shinskey ${ }^{[14]}$ ajusta las constantes de las ecuaciones de sintonización de Ziegler y Nichols para minimizar el criterio IAE; Chidamba$\mathrm{ra}^{[7]}$ utiliza un procedimiento iterativo basado en las mismas ecuaciones de Ziegler y Nichols, pero evitando tener que llevar el sistema al límite de la estabilidad; mientras que Aström et al.${ }^{[5]}$ lo adapta para la sintonización de controladores $P I$ y PID de dos grados de libertad.

\section{PRUEBAS COMPARATIVAS POR SIMULACIÓN}

La bondad de las técnicas de sintonización, descritas anteriormente, fueron evaluadas mediante la simulación digital tanto de las plantas de prueba como de los controladores y se utilizó el programa VisSim $^{\mathrm{TM}} 3.0^{[16]}$.

\subsection{Planta de prueba}

Se seleccionó como planta para realizar la comparación de los métodos de sintonización de controladores, un sistema de tercer orden sobre amortiguado más tiempo muerto dado por la función de transferencia:

$$
G_{p}(s)=\frac{e^{-0.1 s}}{(s+1)(0.5 s+1)(0.25 s+1)}
$$

Es una pequeña variante de la planta de prueba Tipo 2, sugerida por Aström y Hägglund,[5] dada por:

$$
\begin{array}{r}
G_{p 2}(s)=\frac{1}{(s+1)(\alpha s+1)\left(\alpha^{2} s+1\right)\left(\alpha^{3} s+1\right)} \\
\alpha==0.1,0.2,0.5,1.0
\end{array}
$$

Se tomó el valor de $\alpha=0.5$, y el polo más rápido de la planta se reemplazó por un tiempo muerto puro de 0.1 unidades de tiempo. Esto último 
permitió investigar el efecto que el tiempo muerto tiene sobre la respuesta del sistema de control, se supuso que la unidad de tiempo era el minuto.

\section{2 Índices y criterios de desempeño}

Para evaluar el comportamiento dinámico de los sistemas de control y comparar la bondad de los métodos de sintonización estudiados, se tomaron los siguientes índices:

- $\quad$ Error máximo

Se definió como uno de los criterios de desempeño el Porcentaje de Error Máximo (PEM), dado por:

$$
\frac{P E M=100|y(\max )-r(t)|}{r(t)}
$$

en el caso de los sistemas que operan como servomecanismo. También se definió como

$P E M=100 \mid y(t)_{\max }$

en el caso de los sistemas operando como reguladores.

\section{- Tiempo de asentamiento}

Se seleccionó como otro de los criterios de desempeño el Tiempo de Asentamiento $\left(\mathrm{Ta}_{2}\right)$. Es el tiempo requerido por el sistema para que el error entre en una banda del $\pm 2 \%$ del valor deseado:

$T a_{2}=t_{a} \ni|y(t)-r(t)| \leq 0.02 r(t) \forall t \geq t_{a}$

\section{- Integral del error absoluto}

Con base en el error se definió el índice de desempeño:

$I A E=\int_{0}^{\infty}|y(t)-r(t)| d t$

El IAE brinda el área bajo la curva del error, ésta representa la cantidad de material fuera de especificaciones, energía perdida u otra característica indeseada. Si el IAE $\rightarrow 0$, entonces, $y(t) \rightarrow$ $r(t) \forall t$, si el control fuese perfecto.
Aunque la magnitud del IAE es una indicación de la bondad del método de sintonización respecto al error, es más útil, para efectos de comparación respecto a otros métodos de sintonización, su valor relativo respecto al valor del IAE que se obtendría si los parámetros del controlador fueran óptimos respecto a ese criterio.

De esta manera, se definió un Índice de Desempeño en el Error (IDE) de un controlador sintonizado con un conjunto de parámetros que produce un valor determinado IAE como:

$I D E=\frac{I A E_{\text {opt }}}{I A E}$

donde $I A E_{\mathrm{opt}}$ es el valor del IAE para el sistema, con el controlador "óptimo" respecto a este criterio.

\section{- Índices de robustez}

Solamente la medición del desempeño del lazo de control no puede constituirse en la forma de evaluar la bondad de los métodos de sintonización de controladores.

El cálculo de los parámetros del controlador se basa en la obtención de información del proceso a controlar en un punto de operación, usualmente mediante la identificación de un modelo de primer o segundo orden más tiempo muerto.

Los parámetros de esos modelos (ganancia, constantes de tiempo y tiempo muerto aparente) dependen del punto en el cual se realizó la identificación. Aunque ésta se realice en el punto de operación más probable del sistema, la naturaleza no lineal intrínseca en los procesos, hace que los valores de estos parámetros cambien, debido al efecto de las perturbaciones o de cambios en el valor deseado.

Una característica, deseada para los métodos de sintonización de controladores, es que el sistema de control conserve una operación estable ante estos cambios en los parámetros de la planta. La variación requerida para llevar el sistema al límite de la estabilidad es una indicación de esta habilidad, que se denomina robustez. 
Dos parámetros de la planta que pueden sufrir variaciones son la ganancia y el tiempo muerto. Los siguientes Índices de Robustez (IRk $\left.k_{p}, I R t_{m}\right)$ fueron definidos respecto a la variación de estos parámetros como:

$$
\begin{gathered}
I R k_{p}=\frac{k_{p u}}{k_{p a}}-1 ; t_{m}=t_{m a} \\
I R t_{m}=\frac{t_{m u}}{t_{m a}}-1 ; k_{p}=k_{p a}
\end{gathered}
$$

en donde los parámetros con subíndice "a" denotan los valores de los parámetros de la planta utilizados en la sintonización del controlador; asimismo, los parámetros con subíndice " $u$ " denotan los valores que llevan el sistema de control al límite de la estabilidad.

Los índices de robustez (24) y (25) son mediciones independientes de las variaciones requeridas en la ganancia y en el tiempo muerto del sistema, para que éste se inestabilice, eso sí, considerando cada uno por separado, puesto que si la variación es simultánea, el sistema se puede volver inestable mucho antes de que los parámetros alcancen los valores establecidos por los índices de robustez.

De esta manera, se definió un Índice de Robustez Absoluta (IRA) dado por la siguiente expresión:

$$
I R A=\min \left(I R k_{p}, I R t_{m}, 0.5\left[I R k_{p}^{2}+I R t_{m}^{2}\right]^{1 / 2}\right)
$$

\section{3 Índice de desempeño de criterio múltiple}

En el punto 5.2 anterior se establecieron varios índices o criterios de desempeño que pueden ser utilizados para medir la bondad relativa de los diferentes métodos de sintonización que se comparan.

A continuación, establecemos un Índice de Desempeño Absoluto (IDA), criterio múltiple que incluye y pondera los criterios de desempeño ya indicados:

$$
\begin{aligned}
I D A_{i}= & \frac{\left[\frac{20}{P E M_{i}}+\frac{T a_{2 \max }}{T a_{2}}+1.5 I D E_{i}+\frac{I R A_{i}}{2 \sqrt{2}}\right]}{4} \\
& \text { para } P E M \geq 20 \\
I D A_{i} & =\frac{\left[1+\frac{T a_{2 \max }}{T a_{2}}+1.5 I D E_{i}+\frac{I R A_{i}}{2 \sqrt{2}}\right]}{4}
\end{aligned}
$$

$$
\text { para } P E M \leq 20
$$

Los coeficientes de ponderación incluidos en el criterio IDA se seleccionaron de tal manera, que el peso relativo de cada criterio sea similar, así:

- Se penalizaron las respuestas que tenían más de un $20 \%$ de error máximo o sea, valores de PEM superiores a 20.

- Como el tiempo de asentamiento del sistema $\mathrm{Ta}_{2}$ no depende solamente de los parámetros del controlador, sino también de las características dinámicas de la planta, le fue asignado un peso unitario, dentro del criterio, al tiempo de asentamiento del método para que produjera una respuesta más lenta, $\mathrm{Ta}_{2 \max }$; así, los demás métodos de sintonización tienen factores superiores a 1 por este concepto.

- Puesto que el índice de desempeño en el error $I D E$ puede presentar un valor máximo de 1 , consideramos que el método de sintonización tiene que producir valores del IAE superiores al $50 \%$ del óptimo para que este componente del criterio presente valores superiores a 1 .

- Debido a que los índices de robustez $I R k_{p}$ y $I R t_{m}$ deben ser, normalmente, superiores a 1 , es decir, el sistema permanece estable ante variaciones independientes de hasta un $100 \%$ en la ganancia o en el tiempo muerto del proceso, el IRA tendría un valor cercano a $0.5 \sqrt{2}$ como mínimo aceptable, y consideramos deseable que el IRA fuera superior a $2 \sqrt{ } 2$. 


\subsection{Identificación de la planta de prueba}

Se obtuvo la curva de reacción de la planta de prueba (17) y se determinaron los valores necesarios para la identificación de modelos de primer y segundo orden más tiempo muerto mediante varios métodos de identificación.
Los parámetros de los modelos se muestran en los Cuadros 3 y 4 que incluyen el valor de la Integral del Error de Predicción Absoluto $(I A E P)$ de cada uno de ellos. La bondad relativa de los modelos identificados concuerda con los resultados obtenidos en otras plantas de prueba $^{[2]}$

Cuadro 3. Modelos de primer orden más tiempo muerto

Método
Alfaro
Ho et al.
Miller
Smith
Vitecková et al.
Ziegler y Nichols

$\begin{array}{cc}\boldsymbol{k}_{\boldsymbol{p}} & \boldsymbol{\tau} \\ 1 & 1,23 \\ 1 & 1,13 \\ 1 & 1,47 \\ 1 & 1,29 \\ 1 & 1,22 \\ 1 & 2,17\end{array}$

$\boldsymbol{t}_{\boldsymbol{m}}$
0,68
0,77
0,49
0,67
0,71
0,49

Cuadro 4. Modelos de segundo orden más tiempo muerto

$\begin{array}{lccccccc}\text { Método } & \boldsymbol{k}_{\boldsymbol{p}} & \tau_{\boldsymbol{I}} & \tau_{\boldsymbol{2}} & \boldsymbol{t}_{\boldsymbol{m}} & \zeta & \omega_{\boldsymbol{n}} & \text { IAEP } \\ \text { Stark } & 1 & - & - & 0,242 & 0,930 & 1,184 & 0,049 \\ \text { Vitecková } & 1 & 0,78 & 0,78 & 0,282 & - & - & 0,026\end{array}$

Cuadro 5. Parámetros óptimos del controlador PID

$\begin{array}{lcccccc}\text { Sistema control } & \text { Kc } & \text { Ti } & \text { Td } & \text { IAE } & \text { Ta2 } & \text { PEM } \\ \text { Servomecanismo } & 3,061 & 1610 & 0,483 & 0,6255 & 2,488 & 9,55 \\ \text { Regulador } & 7,112 & 0,598 & 0,468 & 0,1324 & 3,269 & 12,78\end{array}$

Cuadro 6. Parámetros para el controlador PID-Ideal

$\begin{array}{lccc}\text { Método } & \boldsymbol{K}_{\boldsymbol{c}} & \boldsymbol{T}_{\boldsymbol{i}} & \boldsymbol{T}_{\boldsymbol{d}} \\ \text { Ziegler y Nichols (ZyN) } & 5,314 & 0,980 & 0,245 \\ \text { Cohen y Coon (CyC) } & 2,207 & 1,506 & 0,249 \\ \text { López et al. - IAE (LIAE) } & 2,043 & 0,744 & 0,352 \\ \text { López et al. - ITAE (LITAE) } & 1,951 & 0,717 & 0,294 \\ \text { López et al. - ISE (LISE) } & 2,148 & 0,764 & 0,430 \\ \text { Sung et al. (SungRmo) } & 5,263 & 0,722 & 0,349\end{array}$


Tabla 7. Parámetros para el controlador PID-Serie

$\begin{array}{lccc}\text { Método } & \boldsymbol{K}_{\boldsymbol{c}} & \boldsymbol{T}_{\boldsymbol{i}} & \boldsymbol{T}_{\boldsymbol{d}} \\ \text { Kaya y Sheib - IAE (KSIAEr) } & 1,314 & 0,829 & 0,480 \\ \text { Kaya y Sheib - ITAE (KSITAEr) } & 1,172 & 0,753 & 0,434 \\ \text { Kaya y Sheib - ISE (KSISEr) } & 1,579 & 0,981 & 0,442\end{array}$

Cuadro 8. Parámetros para el controlador PID-Industrial

$\begin{array}{lccc}\text { Método } & \boldsymbol{K}_{\boldsymbol{c}} & \boldsymbol{T}_{\boldsymbol{i}} & \boldsymbol{T}_{\boldsymbol{d}} \\ \text { Kaya y Sheib - IAE (KSIAEr) } & 1,234 & 0,757 & 0,453 \\ \text { Kaya y Sheib - ITAE (KSITAEr) } & 0,992 & 0,739 & 0,467 \\ \text { Kaya y Sheib - ISE (KSISEr) } & 1.574 & 0.866 & 0.450\end{array}$

Se utilizaron los parámetros del modelo de primer orden más tiempo muerto identificado por el método de la tangente $(\mathrm{ZyN})$ en las ecuaciones de Ziegler y Nichols y los identificados por el método de dos puntos de Ho en los demás casos, con excepción del método de sintonización de Sung que requiere un modelo de segundo orden más tiempo muerto.

\subsection{Parámetros óptimos del controlador}

Para poder establecer el Índice de Desempeño en el Error IDE de cada uno de los métodos de sintonización, tal como se indicó en 5.2 y 5.3, se obtuvieron los conjuntos de parámetros $\left(\mathrm{K}_{\mathrm{c}}\right.$, $\mathrm{T}_{\mathrm{i}}, \mathrm{T}_{\mathrm{d}}$ ) que minimizan el criterio IAE para el sistema que opera tanto como servomecanismo y como regulador.

Los resultados de la optimización se muestran en el Cuadro 5.

\subsection{Parámetros de los controladores}

Los parámetros que se calcularon con los métodos de sintonización descritos en 3.0, aplicables a un controlador PID-Ideal, se muestran en la Cuadro 6. y los correspondientes a controladores PID-Serie o PID-Industrial en los Cuadros 7 y 8 , respectivamente.

\subsection{Resultados de la simulación}

Los controladores PID fueron sintonizados con los parámetros calculados en 5.6. Se obtuvo la respuesta del sistema de control a un cambio de escalón en la perturbación, y se determinaron los valores de las variables necesarias para el cálculo de los índices de desempeño, establecidos en 5.2 y 5.3 .

Los resultados obtenidos se muestran en los Cuadros 9, 10 y 11.

Las curvas de respuesta de los diferentes métodos de sintonización se muestran en las Figuras $2,3,4$ y 5 .

Cuadro 9. Índices de desempeño, controlador PID-Ideal

$\begin{array}{lcccccccc}\text { Método } & \boldsymbol{P E M} & \boldsymbol{T a}_{\boldsymbol{2}} & \boldsymbol{I A E} & \boldsymbol{I D E} & \boldsymbol{I R k}_{\boldsymbol{p}} & \boldsymbol{I R t}_{\boldsymbol{m}} & \text { IRA } & \text { IDA } \\ \text { ZyN } & 19,39 & 3,80 & 0,262 & 0,505 & 1,22 & 1,45 & 0,95 & 1,23 \\ \text { CyC } & 32,52 & 5,02 & 0,682 & 0,194 & 4,60 & 6,80 & 4,10 & 1,12 \\ \text { LIAE } & 30,23 & 6,69 & 0,643 & 0,206 & 4,65 & 4,65 & 3,29 & 0,94 \\ \text { LITAE } & 31,76 & 6,81 & 0,728 & 0,182 & 5,05 & 4,00 & 3,22 & 0,90 \\ \text { LISE } & 28,34 & 5,16 & 0,577 & 0,230 & 3,80 & 5,40 & 3,30 & 1,08 \\ \text { SungR } & 17,40 & 2,87 & 0,202 & 0,656 & 1,18 & 1,68 & 1,03 & 1,52\end{array}$


Cuadro 10. Índices de desempeño, controlador PID-Serie

$\begin{array}{lllllllll}\text { Método } & \boldsymbol{P E M} & \boldsymbol{T a}_{\boldsymbol{2}} & \boldsymbol{I A E} & \boldsymbol{I D E} & \boldsymbol{I R k}_{\boldsymbol{p}} & \boldsymbol{I R t}_{\boldsymbol{m}} & \boldsymbol{I R A} & \text { IDA } \\ \text { KSIAEr } & 33,47 & 3,76 & 0,633 & 0,209 & 4,40 & 7,10 & 4,18 & 1,31 \\ \text { KSITAEr } & 35,59 & 4,75 & 0,698 & 0,190 & 5,00 & 7,20 & 4,38 & 1,16 \\ \text { KSISEr } & 39,34 & 6,09 & 1,054 & 0,126 & 4,00 & 6,60 & 3,86 & 0,96\end{array}$

Cuadro 11. Índices de desempeño, controlador PID-Industrial

$\begin{array}{lcccccccc}\text { Método } & \boldsymbol{P E M} & \boldsymbol{T a}_{\boldsymbol{2}} & \boldsymbol{I A E} & \boldsymbol{I D E} & \boldsymbol{I R k}_{\boldsymbol{p}} & \boldsymbol{I R t}_{\boldsymbol{m}} & \boldsymbol{I R A} & \boldsymbol{I D A} \\ \text { KSIAEr } & 34,44 & 3,56 & 0,654 & 0,202 & 460 & 7,00 & 4,19 & 1,33 \\ \text { KSITAEr } & 37,77 & 3,97 & 0,797 & 0,166 & 5,80 & 8,70 & 5,23 & 1,32 \\ \text { KSISEr } & 31,21 & 3,47 & 0550 & 0,241 & 3,72 & 5,85 & 3,47 & 1,31\end{array}$

Cuadro 12. Posición relativa de los métodos según su IDA

\begin{tabular}{|c|c|c|c|c|c|}
\hline Método & $I D A$ & PEM & $T a_{2}$ & $I D E$ & IRA \\
\hline Sung et al. & 1.52 & $(1)^{*}$ & (1) & (1) & (11) \\
\hline Kaya y Sheib - IAE (ind.) & 1.33 & (9) & (3) & (7) & (3) \\
\hline Kaya y Sheib - ITAE (ind) & 1.32 & (11) & (6) & (11) & (1) \\
\hline Kaya y Sheib - IAE (serie) & 1.31 & (8) & (4) & (5) & (5) \\
\hline Kaya y Sheib - ISE (ind.) & 1.31 & (5) & (2) & (3) & (7) \\
\hline Ziegler y Nichols & 1.23 & (2) & (5) & (2) & (12) \\
\hline Kaya y Sheib - ITAE (ser) & 1.16 & (10) & (7) & (9) & (2) \\
\hline Cohen y Coon & 1.12 & (7) & (8) & (8) & (4) \\
\hline López et al. - ISE & 1.08 & (3) & (9) & (4) & (8) \\
\hline Kaya y Sheib - ISE (ser.) & 0.96 & (12) & (10) & (12) & (6) \\
\hline López et al. - IAE & 0.94 & (4) & (11) & (6) & (9) \\
\hline López et al. - ITAE & 0.90 & (6) & (12) & (10) & (10) \\
\hline
\end{tabular}

* Los números en paréntesis indican la posición relativa del método respecto a cada uno de los criterios

\subsection{Análisis de los resultados}

En 5.3 establecimos un Índice de Desempeño Absoluto (IDA), de esta forma incorporamos en un solo factor de evaluación diferentes características de la respuesta temporal del sistema posibles de medir, tales como el error máximo, el tiempo de asentamiento y la integral del valor absoluto del error, así como la robustez del conjunto de parámetros calculados. Por esta razón en el Cuadro 12 se muestran los métodos estudiados, que ordenamos, según el valor del IDA obtenido con cada uno de ellos y la posición relativa, según cada criterio de evaluación individual.

Si se considera cada criterio en forma individual, vemos que el criterio del PEM, menor al $20 \%$, solamente lo cumplen dos métodos: Ziegler y Nichols y Sung; sin embargo, es necesario destacar que en el caso de los reguladores, siempre habrá una desviación máxima inicial, a diferencia de los servomecanismos, donde la respuesta puede ser sobre amortiguada sin tener sobrepaso. 
La respuesta con el menor tiempo de asentamiento, $\mathrm{T}_{\mathrm{a} 2}$, se logra con el método de Sung, así como con varios de los ajustes de Kaya y Sheib. Las respuestas más lentas las producen los ajustes de López.

El criterio establecido, según el cual el IAE sea como máximo un $50 \%$ mayor que el mínimo, es decir, un $I D E$ mayor a 0.67 , no lo cumple ninguno de los métodos; no obstante, el método de
Sung está muy cerca de este valor. Es importante hacer notar que aunque varios de los métodos de sintonización, como el de López (LIAE) y los de Kaya y Sheib (KSIAE), se supone que fueron optimizados para este criterio, muchos distan bastante de serlo. El valor IDE para el óptimo es 1 ; puesto que estos métodos tienen un valor para este criterio de 0,2 o menor, significa que producen una $I A E$ cinco veces mayor que el mínimo.

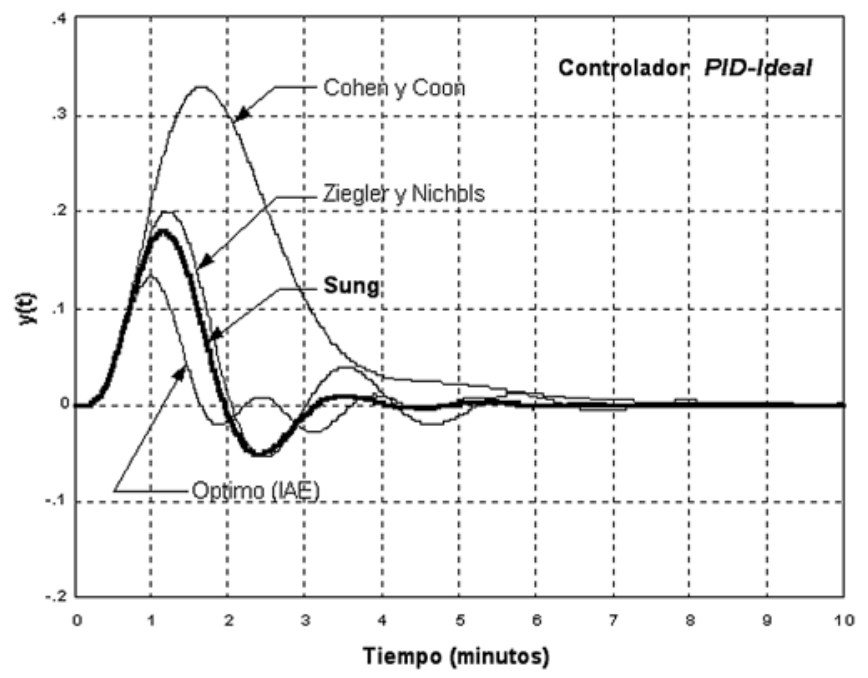

Figura 2. Respuesta a un cambio en la perturbación

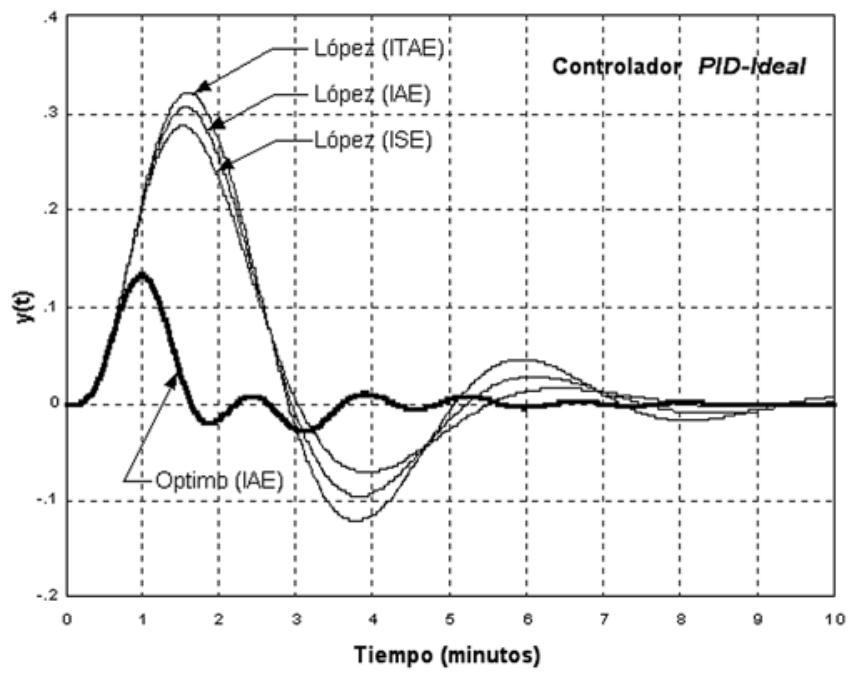

Figura 3. Respuesta a un cambio en la perturbación 


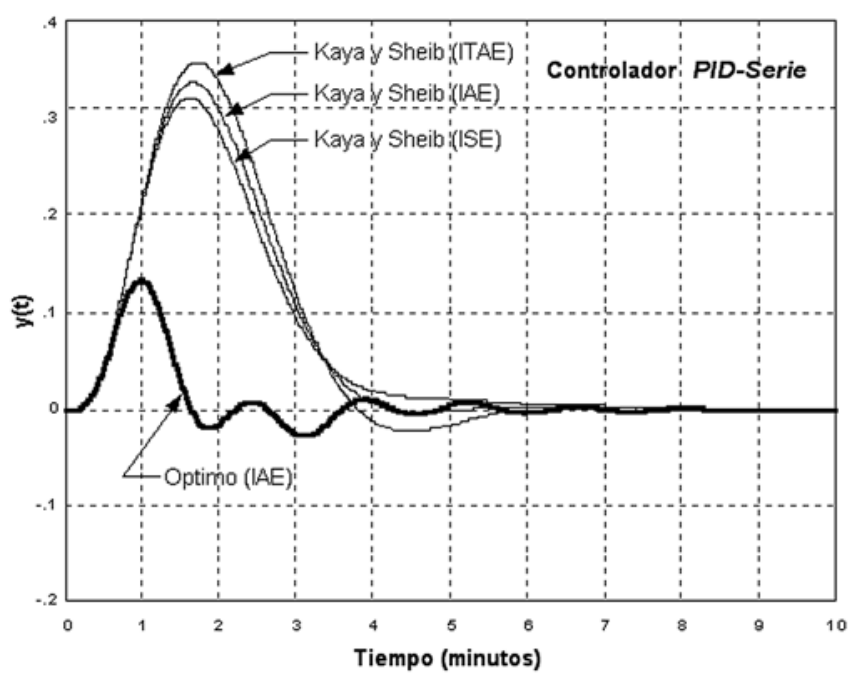

Figura 4. Respuesta a un cambio en la perturbación

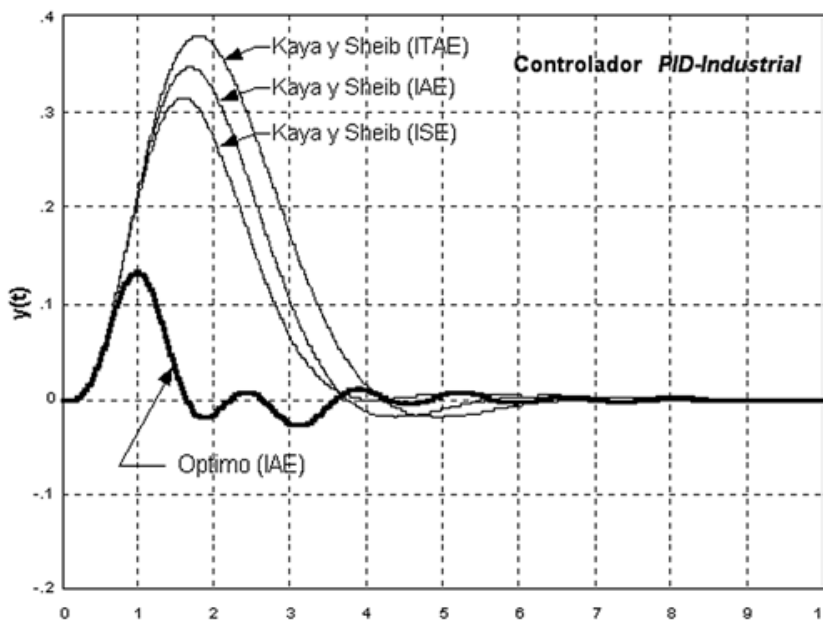

Figura 5. Respuesta a un cambio en la perturbación

Si se comparan los valores del $I D E$ del método de López IAE y el de Kaya y Sheib IAE para el controlador PID Serie e Industrial, vemos que tienen magnitudes similares.

El criterio, según el cual el IRA es superior a $2 \sqrt{ } 2$, lo cumplen diez de los doce métodos. Esto indica que con excepción de los métodos de Ziegler et al. y Sung, los demás son suficientemente robustos.

Al considerar los criterios en forma simultánea, encontramos que no hay ningún método que los cumpla totalmente. En primer lugar, en todos los criterios excepto el de robustez, está el método de Sung. Su índice, $I R A=1,30$, es bastante inferior al valor deseado de 2.82 , pero sus índices de robustez individuales, $I R k_{p}=1,18$ y $I R t_{m}=1,68$, son superiores al valor mínimo aceptable de 1 .

Un aspecto que se debe destacar es el desempeño del método de Ziegler y Nichols, que ocupa posiciones entre los primeros lugares en los diferentes criterios de desempeño excepto en la robustez. La fragilidad de este método es conocida y los resultados lo demuestran. 
Los resultados anteriores indican que se deberá conciliar los diferentes criterios de desempeño en el momento de seleccionar el método de sintonización.

\section{CONCLUSIONES}

De los resultados obtenidos con los métodos de sintonización para reguladores, se puede concluir que si lo que se desea es la respuesta más rápida con el menor error máximo posible, el método de Sung et al. es el mejor, aunque su robustez es bastante baja en comparación con los otros métodos. Las curvas de respuesta, en la Figura 2, muestran claramente la bondad de este ajuste y cuán cercana está la respuesta del método de Ziegler y Nichols.

Buscando un balance en el cumplimiento de los criterios de desempeño establecidos, consideramos que los métodos de Kaya y Sheib IAE, para el controlador PID Industrial o el Serie, son bastante más robustos que los anteriores.

Finalmente, si la robustez no es la mayor preocupación, el método de Sung sería la mejor opción.

\section{BIBLIOGRAFÍA}

1. Alfaro, V.M.; ¿Son todos los controladores PID iguales?, Rev. Ingeniería, San José, Costa Rica, Vol. 3, No 1, 1993.

2. Alfaro, V.M.; Identificación de procesos sobre amortiguados utilizando técnicas de lazo abierto, Rev. Ingeniería, San José, Costa Rica, Vol. 11, N² 2, 2001.

3. Alfaro, V.M.; Identificación de procesos sobre amortiguados utilizando técnicas de lazo cerrado, Rev. Ingeniería, San José, Costa Rica, Vol. 11, N² 2, 2001.

4. Aström, K.J. y T. Hägglund; Automatic Tuning of PID Controllers, Research Tri-angle Park, NC. EUA, Instrument Society of America, 1988.
5. Aström, K.J.; C.C. Hang, P. Person y W.K. Ho - Towards intelligent PID Control, Automatic (UK), Vol. 28 № 1, pág. 1 - 9, 1992.

6. Aström, K.J. y T. Hägglund; Benchmark Systems for PID Control, IFAC Workshop on Digital Control: Past, Present and Future of PID Control, Terrasa, España, Abril $5-7,2000$.

7. Chidambara, M.R.; Chemical Process Control - A new technique for adaptive tuning controller, International Journal of Control (UK), Vol. 12 No 6, pág. 1057 - 1074, 1970.

8. Cohen, G.H. y G.A. Coon; Theoretical Considerations of retarded Control, ASME Transactions (EUA), Vol. 75, pág. 827 - 834, 1953.

9. Corripio, A.B.; Tuning of Industrial Control Systems, ISA - Research Triangle Park, NC, EUA, The Instrumentation, Systems and Automation Society, 2001.

10. Kaya, A. y T.J. Sheib; Tuning of PID Controllers of Different Structures, Con-trol Engineering (EUA), pág. 62 - 65, Dic. 1988

11. López, A.M., J.A. Miller, C.L. Smith y P.W. Murril; Tuning Controllers with Error-Integral Criteria, Instrumentation Technology (EUA), Nov. 1967.

12. O'Dwyer; A Summary of PI and PID Controller Tuning Rules for Processes with Time Delay. Part 1: PI Controller Tuning Rules, IFAC Workshop on Digital Control: Past, Present and Future of PID Control, Terrasa, España, Abril 5-7, 2000.

13. O'Dwyer; A Summary of PI and PID Controller Tuning Rules for Processes with Time Delay. Part 2: PID Controller Tuning Rules, IFAC Workshop on Digital Control: Past, Present and Future of PID Control, Terrasa, España, Abril 5 - 7, 2000. 
14. Shinskey, F.G.; Process Control Systems, Segunda Edición, New York, NY, EUA, McGraw-Hill Book Co., 1979.

15. Sung, S.W., J. O, I.B. Lee, J. Lee y S.H. Yy; - Automatic Tuning of PID Controller using Second-Order plus Time delay Model, Journal of Chemical Engineering of Japan,), Vol. 29 Nº 6, pág. 990 - 999, Japón, 1996.

16. Visual Solutions; VisSim User's Guide Version 3, Westford, Ma., EUA, Visual Solutions, 1999.

17. Witt, S.D. y R.C. Waggoner; Tuning parameters for non-PID three-mode con-trollers, Hydrocarbon Processing (EUA), Vol. $69 \mathrm{~N}^{\mathrm{o}} 6$, Jun. 1999.

18. Ziegler, J.B. y N.B. Nichols; Optimum Settings for Automatic Controls, ASME Transactions (EUA), Vol. 64, pág. 759-768, 1 942.

\section{SIMBOLOGÍA}

$G_{p}(s) \quad$ función de transferencia (modelo)

$G_{c}(s) \quad$ función de transferencia (controlador)

$\mathrm{kp} \quad$ ganancia estática

$\tau, \tau_{1}, \tau_{2} \quad$ constantes de tiempo

$t_{m} \quad$ tiempo muerto aparente

$\omega_{\mathrm{n}} \quad$ frecuencia natural

$\zeta \quad$ razón de amortiguamiento $s \quad$ variable compleja

$K_{c} \quad$ ganancia del controlador

$T_{\mathrm{i}} \quad$ tiempo integral

$T_{\mathrm{d}} \quad$ tiempo derivativo

$\mathrm{K}_{\mathrm{cu}}, T_{\mathrm{u}} \quad$ parámetros últimos

$r(t) \quad$ valor deseado

$y(t) \quad$ variable controlada

$z(t) \quad$ perturbación

\section{NOTAS}

'en el presente artículo se restringe la presentación de las ecuaciones para el cálculo de los parámetros a los controladores PID solamente, para las ecuaciones correspondientes a otros controladores $(P, P D$ o $P I)$ referirse a los artículos originales en la bibliografía

\section{SOBRE EL AUTOR}

\section{Víctor M. Alfaro Ruíz}

Profesor asociado, Departamento de Automática, Escuela de Ingeniería Eléctrica, Universidad de Costa Rica

Apartado postal 2-10, 2060 UCR, San José, Costa Rica

Tel. 207-4472, Fax. 207-4139, correo electrónico: valfaro@eie.ucr.ac.cr

El presente trabajo fue realizado como parte del proyecto $N^{o}$ 731-A0-169 inscrito en la Vicerrectoría de Investigación de la Universidad de Costa Rica. 